\title{
Survey Methods for Assessment of Citrus tristeza virus Incidence in Urban Citrus Populations
}

G. Hughes, Institute of Ecology and Resource Management, University of Edinburgh, Edinburgh, EH9 3JG, UK;
T. R. Gottwald, USDA ARS, U.S. Horticultural Research Laboratory, Fort Pierce, FL 34945; and K. Yamamura,
Laboratory of Population Ecology, National Institute of Agro-Environmental Sciences, Tsukuba 305-8604, Japan

\section{ABSTRACT}

Hughes, G., Gottwald, T. R., and Yamamura, K. 2002. Survey methods for assessment of Citrus tristeza virus incidence in urban citrus populations. Plant Dis. 86:367-372.

This article concerns survey methodology for pathogens in urban citrus populations, motivated in particular by the need for assessments of Citrus tristeza virus (CTV) incidence. We envisage a large area $R$ not devoted primarily to the commercial cultivation of citrus, that nevertheless has a substantial population of citrus trees. It is desired to sample the citrus population of area $R$ in order to be able to make a statement about the level of infection of the population with CTV, or with particular isolates thereof. We describe a two-stage acceptance sampling scheme in which area $R$ is divided into $N$ sampling units, of which $n$ are inspected. The size of the sampling units, while much smaller than $R$, is still large, so subsampling is carried out, introducing the possibility of misclassification of sampling units. To account for misclassification of sampling units, a larger number must be inspected than if it were assumed that there were no misclassifications. We describe the calculation of sample sizes required for subsampling within sampling units and for the total number of sampling units to be inspected, using parameters that can be adjusted to meet different specified regulatory scenarios.

Additional keywords: hypergeometric distribution, inspection errors, operating characteristic

Citrus tristeza virus (CTV) is one of the most important pathogens affecting citrus and is the focus of wide-ranging control efforts $(4,5)$. The choice of an appropriate CTV control method is dependent on the level of CTV incidence, so accurate survey data on the distribution and incidence of CTV-infected trees are a crucial component of control efforts (5). Hughes and Gottwald have previously discussed the assessment of CTV incidence in commercial citrus groves $(8,9)$ and in citrus nurseries (10). In this article, we are concerned with survey methodology for pathogens in urban citrus populations, motivated in particular by the need for assessments of CTV incidence.

In many places where citrus is cultivated commercially, there are, near the areas of commercial production, urban areas in which citrus may be found (11). In Florida, for example, there are large numbers of various types of citrus trees in metropolitan Miami and the surrounding areas, grown either as ornamentals or for dooryard fruit production. The importance of this for

Corresponding author: G. Hughes

E-mail: ghughes@srv0.bio.ed.ac.uk

Accepted for publication 21 November 2001.

Publication no. D-2002-0129-01R

This article is in the public domain and not copyrightable. It may be freely reprinted with customary crediting of the source. The American Phytopathological Society, 2002. neighboring areas of commercial citrus production is twofold. First, the urban citrus represents a potential reservoir of pathogens and vectors that may threaten commercial groves in the short term. Second, the existence of urban citrus alongside other types of plants grown for similar purposes represents an opportunity for virus ingress into citrus (6), providing a longer-term threat. The focus of the present article is thus on the prevention of damage increase where CTV incidence is low. In such circumstances, the opportunity exists to suppress further natural spread of the virus and so avoid an epidemic (5).

The scenario we envisage is that of a large area $R$ not devoted primarily to the commercial cultivation of citrus, that nevertheless has a substantial population of citrus trees. It is desired to sample the citrus population of area $R$ in order to be able to make a statement about the level of infection of the population with CTV, or with particular isolates thereof. Sampling is carried out as follows. $R$ is divided into $N$ sampling units, of which $n$ are inspected. The sampling units, while much smaller than $R$, are still large, so subsampling is carried out. In the $i$ th sampling unit selected for inspection $(i=1, \ldots, n)$, there are $M_{i}$ citrus trees, of which $m_{i}$ will be inspected.

If the true incidence of CTV infection in $R$ is other than very low, the mean incidence and its standard error can be estimated using the appropriate formulae for two-stage cluster sampling for proportions
(1). Instead, we are concerned with the case where the true incidence of CTV infection is very low, such that the outcome of sampling might be that estimated mean incidence is zero. Our assumption is that if CTV infection is actually detected by sampling in this situation, preordained regulatory action will be invoked. The nature and extent of that regulatory action are not topics addressed by this article (but see [4,5] for further discussion). However, if all the sampling units are CTV-negative, it is desirable to be able to make a statement that there is only a (small) specified probability that CTV infection exceeds a (low) specified level of incidence. The question we address here is how to calculate $n$ and $m_{i}$ to meet these specifications.

\section{MATERIALS AND METHODS}

Terminology. The sampling units in area $R$ are referred to as "sites." "Sampling" refers to the inspection of sites. "Subsampling" refers to the inspection of trees within sites.

Subsampling trees within sites. In the $i$ th site, there are $M_{i}$ citrus trees. The incidence of infection is $p_{i}=d_{i} / M_{i}$, where $d_{i}$ is the number of infected trees. Subsampling consists of selecting a simple random sample (SRS) of $m_{i}$ trees. It is assumed that a "gold-standard" assay is used for virus testing at the individual tree level (i.e., testing is-or is assumed to be-errorfree). The assumption that a gold-standard assay is used also applies when virus testing involves Dorfman screening (group testing) (3).

The hypergeometric probability distribution of $X_{i}$, the number of infected trees in the subsample, is given by

$$
\begin{gathered}
\operatorname{Pr}\left(X_{i}=x\right)=\frac{\left(\begin{array}{l}
d_{i} \\
x
\end{array}\right) \cdot\left(\begin{array}{l}
M_{i}-d_{i} \\
m_{i}-x
\end{array}\right)}{\left(\begin{array}{l}
M_{i} \\
m_{i}
\end{array}\right)} x=a, \ldots, b \\
a=\max \left(0, m_{i}-M_{i}+d_{i}\right), b=\min \left(m_{i}, d_{i}\right)
\end{gathered}
$$

The probability of obtaining $c$ or fewer infected trees in a subsample when the actual incidence of infection $p_{i}=d_{i} / M_{i}, d_{i}=$ $0,1, \ldots, M_{i}$, is given by $\operatorname{Pr}\left(X_{i} \leq c\right)=$ $H\left(M_{i}, m_{i}, d_{i}, c\right)$ where $H\left(M_{i}, m_{i}, d_{i}, c\right)=$ $\sum_{x=0}^{c} \operatorname{Pr}\left(X_{i}=x\right)$ and $\operatorname{Pr}\left(X_{i}=x\right)$ is based on equation 1. This equation is referred to as the operating characteristic (OC) function.

Generally, the OC function may be used either to describe the performance of a sampling scheme or, alternatively, to calcu- 
late the sample size required in order to achieve a specified performance. In the case of the current application, the required performance of subsampling can be defined by specifying a point on the $\mathrm{OC}$ function, such that $\operatorname{Pr}\left(X_{i} \leq c\right)=\zeta$ is the probability of a decision, based on subsampling, that a site is infection-free, when the actual (non-zero) incidence of infection is $p_{\zeta}=d_{\zeta, i} / M_{i}$, where $d_{\zeta, i}$ is the corresponding number of infected trees in the $i$ th site. The required minimum subsample size $m_{i}$ is then found from numerical solution of

$$
\zeta=H\left(M_{i}, m_{i}, d_{\zeta, i}, 0\right)
$$

Now, for any chosen $p_{\zeta}$ and $\zeta$ and a given $M_{i}$, a value of $m_{i}$ can be calculated so that, on average, the specified performance is achieved. The choice of $p_{\zeta}$ and $\zeta$ values is discussed further below.

Sampling sites in the area $R$. The area $R$ contains a total of $N$ sites. The sample comprises an SRS of $n$ of these sites. If the CTV status of the sites in the sample was determined without error, the hypergeometric probability distribution of $Y$, the number of infected sites in the sample, would be

$$
\begin{gathered}
\operatorname{Pr}(Y=y)=\frac{\left(\begin{array}{l}
D \\
y
\end{array}\right) \cdot\left(\begin{array}{l}
N-D \\
n-y
\end{array}\right)}{\left(\begin{array}{l}
N \\
n
\end{array}\right)} y=A, \ldots, B ; \\
A=\max (0, n-N+D), B=\min (n, D)
\end{gathered}
$$

in which $D$ is the number of CTV-positive sites in $R$. However, because the CTV status of the sites in the sample is assessed by subsampling trees within sites, the CTV status of a site may be determined incorrectly. Subsampling trees within sites is carried out with subsample sizes $\left(m_{i}\right)$ calculated from equation 2 , such that there is a specified probability $\zeta$ of a decision that a site is infection-free when the actual incidence of infection is $p_{\zeta}$. The OC function on which this calculation is based describes the rate of false negative decisions that a site is infection-free, for different actual levels of incidence of infected trees. The shape of the OC function is such that the false negative rate falls with increasing incidence of infection. The incidence level $p_{\zeta}$ will be regarded as a threshold criterion, set so that the corresponding value of $\zeta$ characterizes the rate at which it is regarded as acceptable to reach false negative decisions. Below this threshold, the false negative rate is regarded as unacceptably high for a reliable statement about the absence of infection. Thus, both $p_{\zeta}$ and $\zeta$ will normally be set at appropriately low values within the constraints of the resources allocated to CTV surveillance. $\zeta$ is actually the maximum rate for false negative decisions, but because one must always be prepared to accept the maximum rate, $\zeta$ is the appropriate value to adopt for the purpose of calculating $n$ and $m_{i}$. The corresponding true positive rate is $1-\zeta$, denoted $P$. It is assumed that no infectionfree sites are wrongly declared infected.

The number of sites in the sample declared infected is denoted $Z$. The conditional probability of declaring $z$ infected sites among the $y$ truly infected sites is then

$$
\operatorname{Pr}(Z=z \mid y)=\left(\begin{array}{l}
y \\
z
\end{array}\right) \cdot P^{z} \cdot(1-P)^{y-z} \quad z=0,1, \ldots, y
$$

and the unconditional probability distribution of $Z$ is

$$
\operatorname{Pr}(Z=z)=\sum_{y=z}^{B} \frac{\left(\begin{array}{l}
D \\
y
\end{array}\right) \cdot\left(\begin{array}{l}
N-D \\
n-y
\end{array}\right)}{\left(\begin{array}{l}
N \\
n
\end{array}\right)} \cdot\left(\begin{array}{l}
y \\
z
\end{array}\right) \cdot P^{z} \cdot(1-P)^{y-z}
$$

$$
z=A, \ldots, B
$$

(with $A$ and $B$ as for equation 3) (15). If $P$ $=1$, the number of infected sites in the sample has a hypergeometric distribution as in equation 3 . If $P<1$, equation 4 describes a "faulty inspection" distribution (12-17) in which there is an acceptable rate $\zeta$ at which infected sites may wrongly be declared infection-free.

When there are no incorrect decisions that a site is infection-free, the probability of obtaining $C$ or fewer infected sites in the sample when the actual incidence of infection is $q=D / N, D=0,1, \ldots, N$, is given by

$\operatorname{Pr}(Y \leq C)=H(N, n, D, C)$

where $H(N, n, D, C)=\sum_{y=0}^{C} \operatorname{Pr}(Y=y) \quad$ and $\operatorname{Pr}(Y=y)$ is based on equation 3. When there is an acceptable false negative rate $\zeta$ $=1-P$ of incorrect decisions that a site is infection-free, the probability of obtaining $C$ or fewer infected sites in the sample when the actual incidence of infection is $q$ $=D / N, D=0,1, \ldots, N$, is given by

$\operatorname{Pr}(Z \leq C)=S(N, n, D, C, P)$

where $S(N, n, D, C, P)=\sum_{z=0}^{C} \operatorname{Pr}(Z=z)$ and $\operatorname{Pr}(Z=z)$ is based on equation 4 .

If there were no incorrect decisions that a site was infection-free, the required performance of the sampling scheme for sites from the area $R$ could be defined by specifying a point on the OC function, such that $\operatorname{Pr}(Y \leq C)=\eta$ is an acceptable probability of a decision, based on sampling, that the area $R$ is infection-free, when the actual (non-zero) incidence of infected sites in $R$ is $q_{\eta}=D_{\eta} / N$, where $D_{\eta}$ is the corresponding number of infected sites. The minimum sample size $(n)$ is then found from numerical solution of

$$
\eta=H\left(N, n, D_{\eta}, 0\right)
$$

Since subsampling within sites introduces inspection errors with an acceptable false negative rate $\zeta$, the required value of $n$ is calculated instead from numerical solution of

$$
\eta=S\left(N, n, D_{\eta}, 0, P\right)
$$

where $P=1-\zeta$.
A binomial approximation. Suppose that $N$ is large enough (so that the sampling fraction $n / N$ is sufficiently small) for the binomial distribution to be used as an approximation of the hypergeometric. Without inspection errors,

$$
\operatorname{Pr}(Y=y)=\left(\begin{array}{l}
n \\
y
\end{array}\right) \cdot q^{y} \cdot(1-q)^{n-y}, y=0,1, \ldots, n
$$

and the usual binomial sample size formula

$$
n=\frac{\ln (\eta)}{\ln \left(1-q_{\eta}\right)}
$$

is applicable. With inspection errors, the probability that all $y$ truly infected sites in the sample of sites are declared infectionfree is $\zeta^{y}$. Hence, the probability that no sites are declared infected is

$$
\operatorname{Pr}(Z=0)=\sum_{y=0}^{n} \zeta^{y} \cdot \operatorname{Pr}(Y=y)=[1-(1-\zeta) \cdot q]^{n}
$$

(using Newton's binomial formula). Denote as $\eta$ the required $\operatorname{Pr}(Z=0)$ when $q=$ $q_{\eta}$, write $\lambda_{\eta}=(1-\zeta) \cdot q_{\eta}$, solve for $n$, and

$n=\frac{\ln (\eta)}{\ln \left(1-\lambda_{\eta}\right)}$

Equation 9 is retrieved when $\zeta=0$.

A check. The following analysis is based on methodology discussed by Winkler (21) and Pham-Gia and Turkkan (18). There is (from the within-site subsampling) an acceptable false negative rate $\zeta$ for decisions that a site is infection-free. It is assumed here that this is the only kind of inspection error, but for completeness the false positive rate for infection-free sites wrongly declared infected is denoted $\delta$ (and in all the calculations below, $\delta=0$ ). The probability of testing positive (including true and false positives), denoted $\lambda$, is related to the true proportion infected, $q$, by

$$
\lambda=q \cdot(1-\zeta)+(1-q) \cdot \delta
$$

The sampling process is Bernoulli in $\lambda$, rather than in $q$ (21).

Suppose that an SRS of $n$ sites is drawn. The maximum likelihood estimate (MLE) of the observed sample proportion infected is denoted $\hat{\lambda}$. It is assumed that the observed sample proportion infected lies between $\delta$ and $1-\zeta$ (see [2] for a discussion on the limitations of this procedure). The MLE of $q$, the true proportion of sites infected, is

$\hat{q}=\frac{\hat{\lambda}-\delta}{1-\zeta-\delta}$. Let $k_{1}=\frac{\delta}{1-\zeta-\delta}$ and $k_{2}=\frac{\zeta}{1-\zeta-\delta}$.

Then $n^{*}$ is defined as

$n^{*}=\frac{n \cdot \hat{q} \cdot(1-\hat{q})}{\left(\hat{q}+k_{1}\right) \cdot\left(1-\hat{q}+k_{2}\right)}$

As long as the initial assumption is satisfied, $n>n^{*}$. Equation 11 shows the relationship between the required sample 
size $(n)$ and the equivalent number of observations $\left(n^{*}\right)$ if there were no errors in the classification of sites.

The quantity $n^{*}$ can be thought of as an effective sample size (2) that takes into account the possibility of misclassifications resulting from the subsampling process. It is of particular interest, therefore, to consider the case of a sample of $n$ observations drawn such that $\hat{\lambda}=q_{\eta}$, since equation 11 may then be used to verify that the effective sample sizes corresponding to required sample sizes calculated from equation 8 (i.e., on the basis of equation 4), with inspection errors, are (at least to a good approximation) the same as the sample sizes calculated from equation 7 (i.e., on the basis of equation 3), without inspection errors.

\section{RESULTS}

The analysis outlined above is illustrated here for an example in which the adopted values of $p_{\zeta}, q_{\eta}$, and $\eta$ are $0.02,0.05$, and 0.1 , respectively. In practice, these values may be adjusted to meet different specified regulatory scenarios.

First, subsampling trees within sites was considered. Figure 1 shows OC curves for various $p_{\zeta}, \zeta$ combinations: $p_{\zeta}=0.02 ; \zeta=$ $0.25,0.2,0.15,0.1,0.05$. As the acceptable probability of a decision, based on subsampling, that a site is infection-free $(\zeta)$ decreases at a given actual incidence of infection $\left(p_{\zeta}\right)$, the performance required of the subsampling scheme is being increased. The subsample sizes $m_{i}$ for population sizes $M_{i}$ required for each of the OC curves shown in Figure 1 are shown in Figure 2. Note that as the performance required of the subsampling scheme is increased, the required subsample sizes increase.

Sampling of sites within area $R$ was then considered. Figure 3 shows OC curves for equation 5 (no incorrect decisions about the CTV status of sites) and equation 6 (acceptable rate of false negative decisions about the CTV status of sites $\zeta=0.05$, $0.10,0.15,0.20,0.25)$. Plotting OC curves based on equations 5 and 6 , using the same values of $N, n$, and $C$, and values of $P<1$ in equation 6 , showed that the OC curve based on equation 6 is shifted to the right relative to that for equation 5 (see also 20). The effect of introducing the possibility of false negative decisions into the classification of sites (due to subsampling within sites) is to increase $\eta$ above the nominal value $(\eta=0.1)$ at the adopted $q_{\eta}$ (Fig. 3).

The OC curve for equation 5 in Figure 3 has the required specification. If there were no incorrect decisions about the CTV status of sites, the number of sites to be inspected $(n)$ in order to meet this specification would be provided by equation 7 . However, because subsampling within sites introduces errors in the classification of sites, the number of sites to be inspected in order to meet this specification is instead

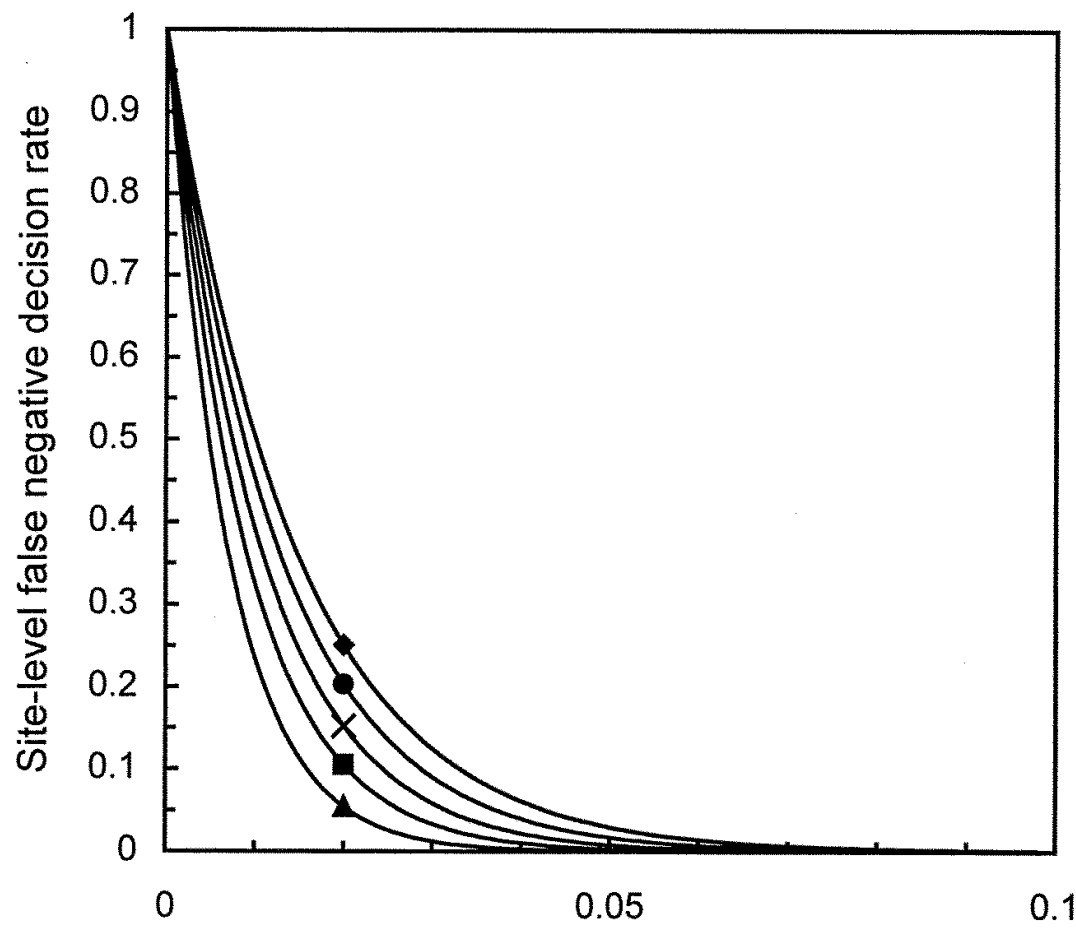

\section{Actual incidence of infected trees}

Fig. 1. Examples of operating characteristic (OC) curves for subsampling trees within sites. These show the probability of a decision that a site is infection-free for different actual levels of incidence of infected trees. The OC curves are characterized by an acceptable false negative decision rate $(\zeta)$ at a particular level of incidence of infected trees $\left(p_{\zeta}\right)$ as follows: $p_{\zeta}=0.02 ; \zeta=0.25(\bullet), 0.20(\bullet), 0.15$

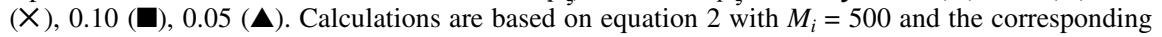
$m_{i}$ values taken from Figure 2.

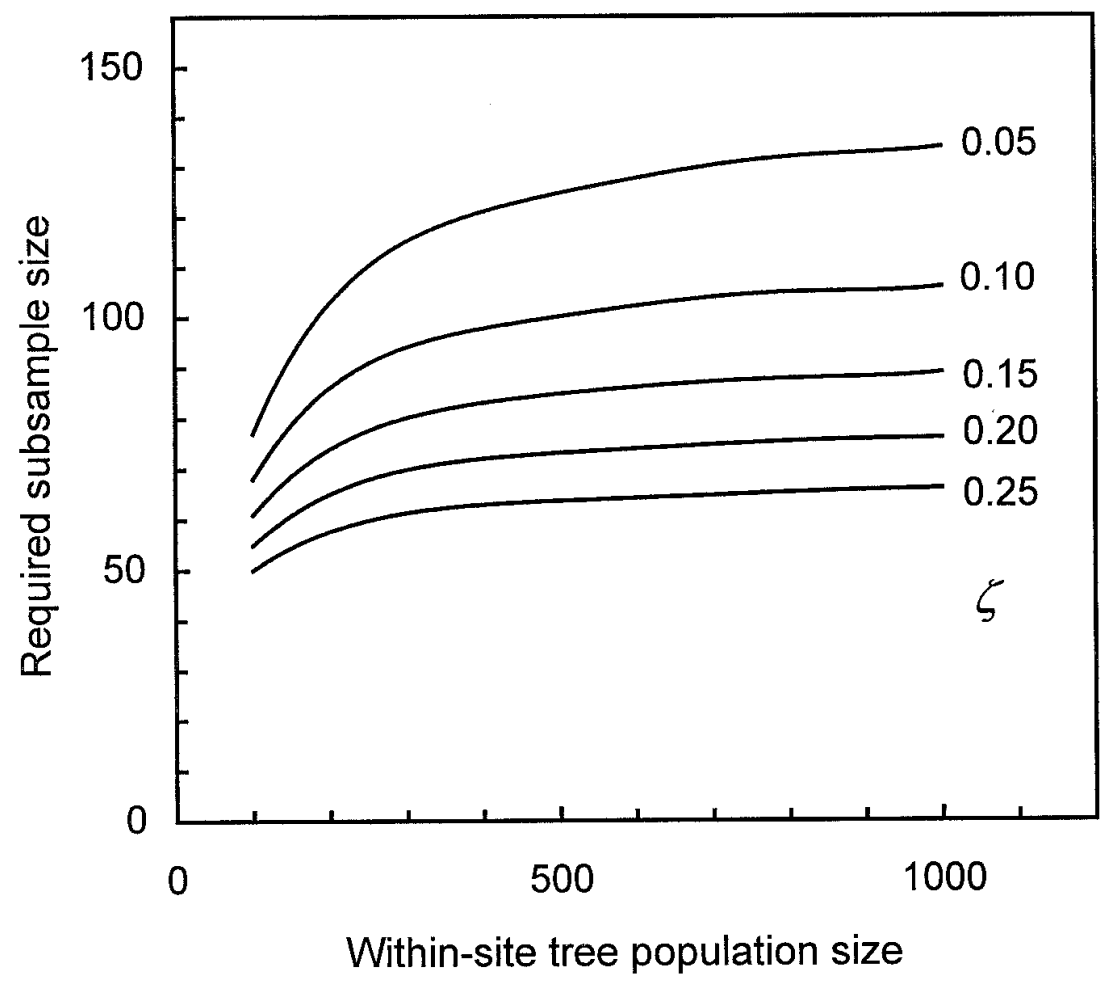

Fig. 2. Relationships between required subsample size $\left(m_{i}\right)$ and within-site tree population size $\left(M_{i}\right)$, calculated from equation 2 , for each of the operating characteristic curves shown in Figure 1 (characterized by $p_{\zeta}=0.02 ; \zeta=0.25,0.20,0.15,0.10,0.05$ ). 
calculated from equation 8 . For same $\eta, N$, $D_{\eta}$, and $C(=0), n$ calculated from equation 8 is larger than $n$ from equation 7 . If $N$ is large enough (so that the sampling fraction $n / N$ is sufficiently small) for the binomial distribution to be used as an approximation of the hypergeometric, the number of sites to be inspected in order to meet the required specification is given by equation 10 (that takes into account errors in the classification of sites) instead of equation 9 (that does not). For both equations 8 and 10 , number of sites to be inspected increases with increasing $\zeta$ (Fig. 4).

As a check on the above analysis, the effective sample sizes $\left(n^{*}\right.$, equation 11$)$ were calculated for values of $n$ derived from equation 8 or equation 10 (as in Figure 4). For these calculations, $\hat{\lambda}=q_{\eta}(=0.05), \zeta=$ $0.05,0.10,0.15,0.20,0.25$, and $\delta=0$. In all cases, the calculated values of $n^{*}$ were, to a very good approximation, the same as the corresponding values of $n$ calculated from equation 7 or equation 9 (Table 1).

\section{DISCUSSION}

We have described a two-stage acceptance sampling scheme in which a large area $R$ is divided into $N$ sites, of which $n$ are inspected. The size of the sites, while much smaller than $R$, is still large, so subsampling is carried out, introducing the possibility of misclassification of sites. To account for misclassification of sites, a larger number of sites ( $n$ calculated from equation 8 or 10) must be inspected than if it were assumed that there were no inspection errors (in which case $n$ would be calculated from equation 7 or 9).

All calculations presented here for subsample size $m_{i}$ use equation 2 , but in practice, it is often more convenient to calculate $m_{i}$ from the approximation formula

$$
m_{i} \approx\left(M_{i}-\frac{d_{\zeta, i}-1}{2}\right) \cdot\left(1-\zeta^{\frac{1}{d_{\zeta, i}}}\right)
$$

(22). Values of $m_{i}$ calculated from equation 12 are slightly conservative (22). If the scenario were such that the area $R$ was made up of a large number of sparsely populated sites, it would probably be sufficient to calculate $n$ and $m_{i}$ from equations 10 and 12 , respectively, thus avoiding the need for iterative solutions to equations. Whether values of $m_{i}$ are calculated from equation 2 or equation 12, values for the $M_{i}$ are required. When these values (the within-site population sizes) are not known, we suggest that the conservative practice of rounding values for $M_{i}$ provided by the assessor, up to the nearest 100 , be used. Other than where the number of trees at a site is small, the required within-site subsample size does not increase markedly with within-site population size (Fig. 2), so

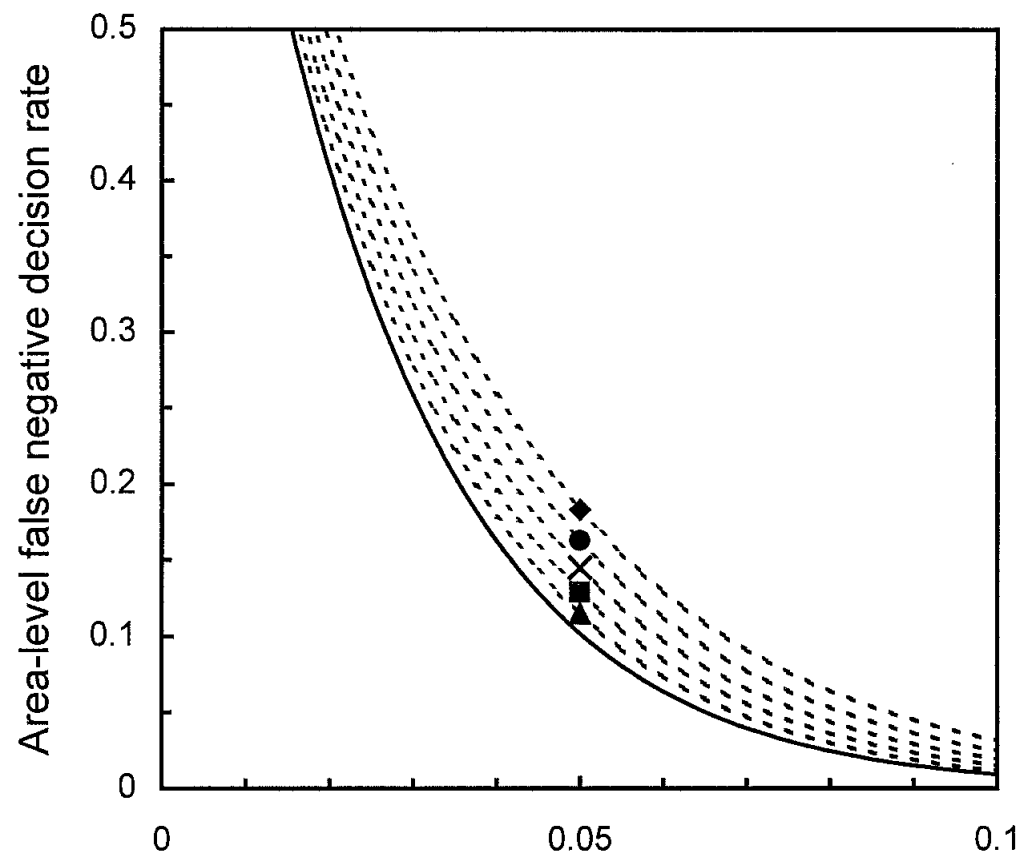

Actual incidence of infected sites

Fig. 3. The solid line represents the operating characteristic (OC) curve for sampling sites in the area $R$, calculated from equation 5 (no incorrect decisions about the Citrus tristeza virus [CTV] status of sites) with $N=1,000, n=43, C=0$. This line shows the probability of a decision that $R$ is infectionfree for different actual levels of incidence of infected sites. The OC curve is characterized by an acceptable false negative decision rate $(\eta)$ at a particular incidence of infected sites $\left(q_{\eta}\right)$ as follows: $q_{\eta}=0.05, \eta=0.1$. The dashed lines (---) show the effect on the OC curve of subsampling within sites, introducing an acceptable false negative decision rate for the CTV status of sites of $\zeta=0.25$ $(\bullet), 0.20(\bullet), 0.15(\times), 0.10(\mathbb{\square}), 0.05(\boldsymbol{\Delta})$. These are calculated from equation 6 with $P=1-\zeta$, other parameters as given above.

this practice should not lead to a large increase in the number of trees to be inspected.

The value $\zeta$ is the acceptable false negative rate for decisions that an infected site is infection-free. Within a site, the number of trees to be inspected $\left(m_{i}\right)$ decreases as $\zeta$ increases (Fig. 2), all other things being equal. However, the number of sites to be inspected $(n)$ increases with increasing $\zeta$ (Fig. 4). Various combinations of $n$ and $m_{i}$ may be used to achieve a stated overall objective defined by the choice of $q_{\eta}$ and $\eta$. If there are cost considerations related to sampling within/between sites, it may be possible to identify an optimal cost combination of $n$ and $m_{i}$. The total number of trees to be inspected is $\sum_{i=1}^{n} m_{i}$.

The choice of $p_{\zeta}$ and $\zeta$ values is a fundamental aspect of constructing the survey. In the current article, dealing with a single pathogen, we have fixed $p_{\zeta}$ and controlled the level of risk by changing $\zeta$. Where this type of survey methodology is adopted for a wider range of pests, an alternative approach is to adopt a fixed value of $\zeta$ and control the level of risk by changing the $p_{\zeta}$ value (see Figure 3 in literature citation 22). A smaller $p_{\zeta}$ may be then used for serious pests and a rather larger $p_{\zeta}$ for nonserious pests.

The analysis outlined above requires that the subsamples of trees within sites are SRSs of the within-site tree populations, and that the sample of sites is an SRS from the population of sites in the area $R$. Simple random sampling implies that infected and uninfected units have the same probability of being inspected. At the withinsite level, an SRS can be achieved if trees are selected at random, even if the spatial pattern of infected trees is aggregated (although at the very low levels of incidence at which the sampling scheme described here is intended for use, trying to characterize nonrandom patterns of CTVinfected trees would be wasted effort). Practically, however, it may be difficult to achieve a true SRS at the within-site level, given that an assessor will usually need to traverse a path through a site, ending close to the starting point, and that along the way there may be unforeseen problems of access to private property for the purpose of tree inspection. If, in practice, subsampling is carried out according to some (perhaps approximately) systematic procedure, the minimum requirement is that an element of randomization is introduced to the selection of the starting point. There is, on the other hand, a good chance to select a true SRS of sites from the area $R$, because this can be done at the outset of sampling, based on a map of the area.

Sampling of citrus in the metropolitan Miami area is currently carried out to detect citrus canker (caused by Xanthomonas axonopodis pv. citri) $(7,19)$. The sentinel grid scheme in use for citrus canker detection has different objectives from the type 
of sampling outlined in the present article; specifically, to detect rapidly the spread of new canker infections during an epidemic. The two-stage acceptance sampling scheme for CTV infection described here is applicable in routine surveillance of large areas where the incidence of CTV infection is very low. The area is divided

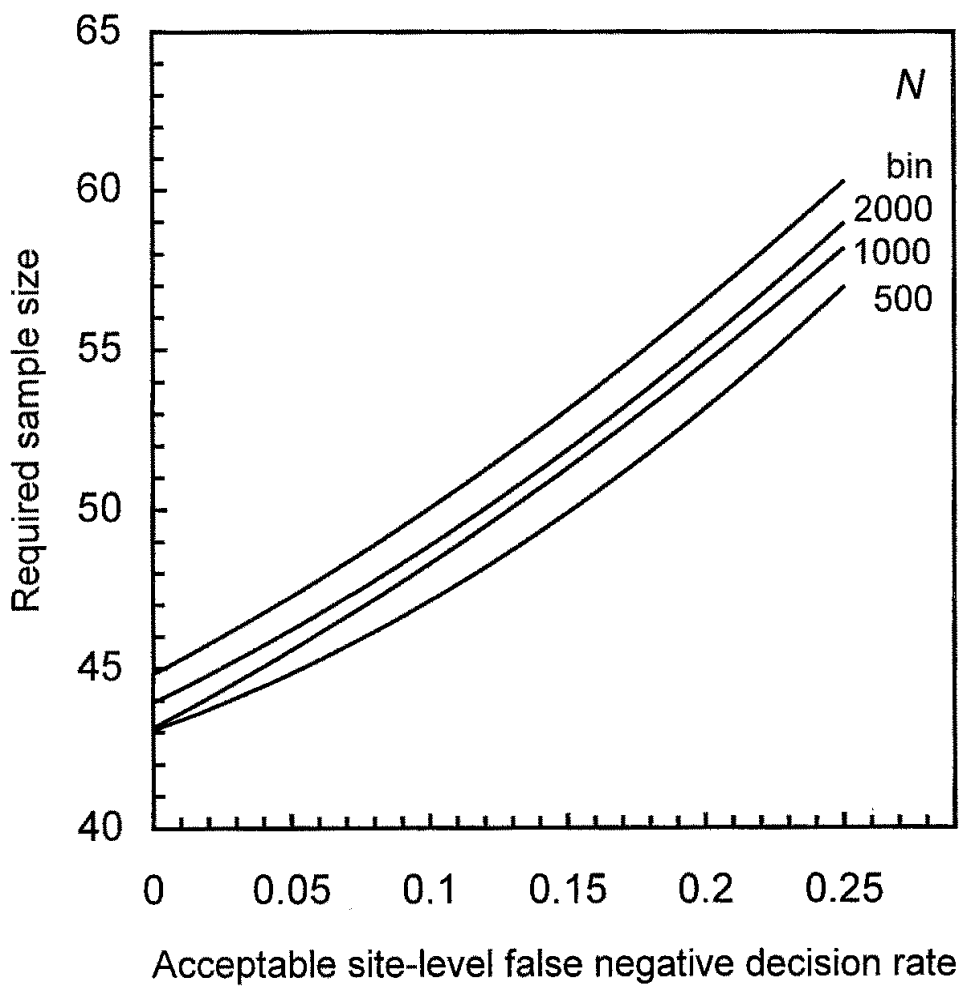

Fig. 4. Relationships between the required sample size ( $n$, the number of sites to be inspected) and the acceptable rate of false negative decisions about the Citrus tristeza virus (CTV) status of sites $(\zeta)$ for a range of population sizes ( $N$, the number of sites). For given $\zeta$ and $N$, a sample size of $n$ ensures that the performance of sampling is described by an operating characteristic (OC) curve characterized by an acceptable false negative decision rate for the CTV status of area $R(\eta)$ at a particular incidence of infected sites $\left(q_{\eta}\right)$. The adopted values of $q_{\eta}$ and $\eta$ are 0.05 and 0.1 , respectively, so the performance of sampling is described by the OC curve shown as the solid line in Figure 3. For $N=500,1,000$, and 2,000, the calculations are based on either equation $7(\zeta=0)$ or equation $8(\zeta>0)$. Where $N$ is denoted "bin," this indicates that the number of sites is large enough for the binomial distribution to be used as an approximation of the hypergeometric, in which case the calculations are based on either equation $9(\zeta=0)$ or equation $10(\zeta>0)$.

Table 1. Effective sample sizes $(n *)$ calculated from equation 11, for actual sample sizes $\left(n^{\mathrm{a}}\right)$ calculated from equation 8 or equation 10 taking inspection errors into account, compared with the corresponding sample sizes $\left(n^{\text {b }}\right)$ calculated from equation 7 or equation 9 that do not take inspection errors into account

\begin{tabular}{llcc}
\hline Population size, $\boldsymbol{N}$ & $\boldsymbol{n}^{\mathbf{b}}$ & $(\boldsymbol{\zeta}=\mathbf{0 . 0 5}$ to $\mathbf{0 . 2 5})$ & $\begin{array}{c}\boldsymbol{n}^{\mathbf{a}} \\
(\zeta=\mathbf{0 . 0 5} \text { to 0.25) }\end{array}$ \\
\hline 500 & $43^{\mathrm{c}}$ & $45-57^{\mathrm{d}}$ & $42-43^{\mathrm{e}}$ \\
1,000 & $43^{\mathrm{c}}$ & $46-58^{\mathrm{d}}$ & $43-44^{\mathrm{e}}$ \\
2,000 & $44^{\mathrm{c}}$ & $46-59^{\mathrm{d}}$ & $44^{\mathrm{e}}$ \\
bin $^{\mathrm{f}}$ & $45^{\mathrm{g}}$ & $47-60^{\mathrm{h}}$ & $45^{\mathrm{e}}$ \\
\hline
\end{tabular}

a Sample sizes calculated accounting for inspection errors (see Figure $4, \zeta>0$ ).

${ }^{\mathrm{b}}$ Sample sizes calculated without accounting for inspection errors (see Figure $4, \zeta=0$ ).

${ }^{\mathrm{c}}$ Calculated from equation 7 with $\eta=0.1, q_{\eta}=0.05$.

${ }^{\mathrm{d}}$ Calculated from equation 8 with $\eta=0.1, q_{\eta}=0.05, P=1-\zeta$.

${ }^{\mathrm{e}}$ Calculated from equation 11 with $\lambda=q_{\eta}=0.05, \hat{q}=(\lambda-\delta) /(1-\zeta-\delta), k_{1}=\delta /(1-\zeta-\delta)$, and $k_{2}=\zeta /(1-\zeta-\delta)$ (and $\delta=0$ ). If the number of sites is large enough for the binomial distribution to be used as an approximation of the hypergeometric in calculations of sample size (see footnote f), $n$ is obtained from equation 10 (see footnote h). Otherwise $n$ is obtained from equation 8 (see footnote d).

${ }^{\text {f }}$ Where $N$ is denoted "bin," this indicates that the number of sites is large enough for the binomial distribution to be used as an approximation of the hypergeometric in calculations of sample size. Sample sizes taking inspection errors into account may then be calculated from equation 10 (see footnote h) rather than equation 8 (see footnote d), while sample sizes that do not take inspection errors into account may be calculated from equation 9 (see footnote g) rather than equation 7 (see footnote c).

g Calculated from equation 9 with $\eta=0.1, q_{\eta}=0.05$.

${ }^{\mathrm{h}}$ Calculated from equation 10 with $\eta=0.1, q_{\eta}=0.05, \lambda_{n}=(1-\zeta) \cdot q_{n}$.

into sites, a number of which are inspected by subsampling. If all the inspected sites are found to be CTV-negative, it is desirable to be able to make a statement that there is only a (small) specified probability $(\eta)$ that CTV infection at the site level exceeds a (low) specified level of incidence $\left(q_{\eta}\right)$. The methodology outlined here for calculating the subsample and sample sizes required to meet these specifications is transferable to similar scenarios with other citrus pathogens, and potentially also to some noncitrus pathosystems.

\section{LITERATURE CITED}

1. Cochran, W. G. 1977. Sampling Techniques, 3rd ed. John Wiley \& Sons, New York.

2. Cussens, J. 1996. Effective sample size in a dichotomous process with noise. Commun. Stat. - Theor. Methods 25:1233-1246.

3. Dorfman, R. 1944. The detection of defective members of large populations. Ann. Math. Stat. 14:436-440. (reprinted as Appendix B of literature citation 16)

4. Garnsey, S. M. 1999. Systemic diseases. Pages 95-106 in: Citrus Health Management. L. W. Timmer and L. W. Duncan, eds. American Phytopathological Society, St. Paul, MN.

5. Garnsey, S. M., Gottwald, T. R., and Yokomi, R. K. 1998. Control strategies for citrus tristeza virus. Pages 639-658 in: Plant Virus Disease Control. A. Hadidi, R. K. Khetarpal, and H. Koganezawa, eds. American Phytopathological Society, St. Paul, MN.

6. Garnsey, S. M., and Hilf, M. E. 2000. Assembling clues on the origins and dissemination of Citrus tristeza virus. (Abstr.) Phytopathology 90:S91.

7. Gottwald, T. R., Hughes, G., Graham, J. H., Sun, X., and Riley, T. 2001. The citrus canker epidemic in Florida: The scientific basis of regulatory eradication policy for an invasive species. Phytopathology 91:30-34.

8. Hughes, G., and Gottwald, T. R. 1998. Survey methods for assessment of citrus tristeza virus incidence. Phytopathology 88:715-723.

9. Hughes, G., and Gottwald, T. R. 1999. Survey methods for assessment of citrus tristeza virus incidence when Toxoptera citricida is the predominant vector. Phytopathology 89:487-494.

10. Hughes, G., and Gottwald, T. R. 2001. Survey methods for assessment of Citrus tristeza virus incidence in citrus nurseries. Plant Dis. 85:910-918.

11. Hughes, G., Gottwald, T. R., and Garnsey, S. M. 2001. Development of methods and models and their application to disease problems in the perennial citrus crop system. Pages 307-329 in: Biotic Interactions in PlantPathogen Associations. M. J. Jeger and N. J. Spence, eds. CAB International, Wallingford, UK.

12. Johnson, N. L., and Kotz, S. 1985. Some distributions arising as a consequence of errors in inspection. Nav. Res. Logistics Q. 32:35-43.

13. Johnson, N. L., Kotz, S., and Kemp, A. W. 1992. Univariate Discrete Distributions, 2nd ed. John Wiley \& Sons, New York.

14. Johnson, N. L., Kotz, S., and Rodriguez, R. N. 1985. Statistical effects of imperfect inspection sampling: I. Some basic distributions. J. Quality Technol. 17:1-31.

15. Johnson, N. L., Kotz, S., and Sorkin, H. L. 1980. "Faulty inspection" distributions. Commun. Stat. - Theor. Methods A9:917-922.

16. Johnson, N. L., Kotz, S., and Wu, X. 1991. Inspection Errors for Attributes in Quality Control. Chapman \& Hall, London.

17. Lindsay, B. G. 1985. Errors in inspection: Integer parameter maximum likelihood in a 
finite population. J. Am. Stat. Assoc. 80:879885.

18. Pham-Gia, T., and Turkkan, N. 1992. Information loss in a noisy dichotomous sampling process. Commun. Stat. - Theor. Methods 21:2001-2018.

19. Schubert, T. S., Rizvi, S. A., Sun, X., Gottwald, T. R., Graham, J. H., and Dixon, W.
N. 2001. Meeting the challenge of eradicating citrus canker in Florida - again. Plant Dis. 85:340-356.

20. Suich, R. 1990. The effects of inspection errors on acceptance sampling for nonconformities. J. Quality Technol. 22:314-318.

21. Winkler, R. L. 1985. Information loss in noisy and dependent processes. Pages 559-570 in:
Bayesian Statistics 2. J. M. Bernardo, M. H. DeGroot, D. V. Lindley, and A. F. M. Smith, eds. Elsevier Science Publishers B.V., NorthHolland.

22. Yamamura, K., and Sugimoto, T. 1995. Estimation of the pest prevention ability of import plant quarantine in Japan. Biometrics 51:482490. 